\title{
ESTIMATIVA DA QUANTIDADE DE ESCÓRIA PASSANTE NO VAZAMENTO DO FEA ATRAVÉS DE BALANÇO DE MASSA*
}

\author{
Thales Botelho' \\ Gabriel Evangelista Medeiros ${ }^{2}$ \\ André Luiz Vasconcellos da Costa e Silva ${ }^{3}$ \\ Juliano Graziel Serra ${ }^{4}$
}

\section{Resumo}

Em função das condições desejadas no processamento do aço no Forno Elétrico a Arco (FEA), a escória final tem teor elevado de FeO, contém fósforo e pode ter, ainda, alto teor de alumina. Para controlar o consumo de desoxidantes, formar uma escória adequada no forno panela (FP) e evitar a reversão de fósforo, é importante minimizar a passagem da escória do FEA para a panela, no vazamento. Assim, é essencial conhecer a quantidade de escória passante para o controle e previsão adequados do processamento no FP. Neste trabalho é apresentada uma metodologia para, através de um balanço de massa levando em conta medidas de composição realizadas durante o processo, determinar a quantidade de escória passante e o volume de escória no final do processamento no FP. É empregado um método numérico simples e demonstra-se que os resultados obtidos são suficientemente adequados para melhor conhecimento e controle do processo por parte do engenheiro responsável, possibilitando gerar informações precisas para cálculos de correções (exemplo: formulação de uma nova escória sintética, correção de desgaste de refratários, ajuste de basicidade, etc.).

Palavras-chave: Forno elétrico; Escória; Balanço de massa; Refino secundário.

\section{ESTIMATING THE AMOUNT OF SLAG TRANSFERED FROM EAF TO LADLE DURING TAPPING USING A MASS BALANCE}

\section{Abstract}

In view of the desired slag conditions during processing in the Electric Arc Furnace (EAF), the final slag has a high $\mathrm{FeO}$ content, contains phosphorous and may also have a high alumina content. To control the consumption of deoxidants, create a slag adequate for the processing in the ladle furnace (LF) and avoid phosphorous reversion, it is important to minimize the transfer of EAF slag to the ladle during tapping. Thus, in order to properly control the LF operation and predict its behavior, it is essential to know the amount of slag transferred with the steel during tapping. In the present work we present a methodology to determine the amount of transfer slag as well as the final amount of slag in the ladle. The methodology uses a mass balance based on composition measurements made during the process. A simple numeric method is used and it is shown that the results derived from the model are sufficiently accurate to improve the process control and understanding by the process engineer, providing accurate information to make adjustment calculations (example: formulation of a new synthetic slag, adjustment of refractory erosion and basicity, etc.).

Keyword: Electric furnace; Slag; Mass balance; Ladle furnace.

1 Aluno de graduação em Engenharia Metalúrgica, EEIMVR-UFF, Volta Redonda, RJ, Brasil.

2 Engenheiro de Materiais, Engenheiro de Processos na Aciaria Elétrica Gerdau Cosigua, Rio de Janeiro, RJ, Brasil.

3 Professor Titular, PhD, EEIMVR-UFF, Volta Redonda, RJ, Brasil.

${ }^{4}$ Engenheiro Metalurgista, Chefe dos fornos da Aciaria Elétrica Gerdau Cosigua, Rio de Janeiro, RJ, Brasil. 


\section{INTRODUÇÃO}

O conhecimento e controle da escória, na aciaria, em todas as etapas do processamento do aço, é essencial para controlar a qualidade do produto, o rendimento metálico, rendimento de ligas e a eficiência energética [1].

O nível de entendimento que hoje se dispõe dos processos que ocorrem tanto no FEA [2] como FP [3,4] permitem compreender como as características da escória (composição química, fração líquida, viscosidade, etc) afetam o processo e as características do aço produzido. Isto permite que, presentemente, se projete as escórias de aciaria para as finalidades desejadas.

A escória gerada durante o refino primário no FEA é ajustada com vistas a sua otimização: espumação, oxidação, desfosforação, etc. Estas características, entretanto, não são adequadas para o FP: os elevados teores de $\mathrm{FeO}(20-35 \%)$ podem conduzir ao desgaste acelerado do revestimento refratário da panela e ao consumo elevado de desoxidantes. Além disto, as condições desoxidantes desejadas no FP levam a reversão do fósforo contido na escória de FEA que venha a passar para a panela. Por fim, quando a escória do FEA contém elevados teores de alumina, as inclusões formadas no produto final assim como as propriedades físicas da escória da panela podem se desviar do desejado. Portanto, é importante que se minimize a passagem de escória do FEA para a panela no refino secundário. Embora diversos métodos para detectar o início da passagem de escória para a panela sejam disponíveis (métodos térmicos, magnéticos, etc. [5]) o vazamento sem escória é muito difícil e, devido a formação de vórtices no vazamento requer, mesmo nos fornos de vazamento excêntrico, pelo fundo, a operação com grande quantidade de pé liquido, que pode não ser desejada. Quando há passagem de escória, a condição mais normal, existem grandes dificuldades operacionais para quantificar corretamente a quantidade de escória transferida do FEA para a panela, mas, para isto, a primeira dificuldade é a medição desta quantidade.

Para contornar o problema da dificuldade de medição da quantidade de escória transferida, criou-se um modelo de balanço de massa, focado na escória. O modelo se baseia em que todas as quantidades de materiais que entram e saem do sistema tenham seu peso e composição conhecida. Entretanto, as análises químicas necessárias para o balanço podem introduzir incertezas consideráveis. Para contornar esta dificuldade, o modelo que será apresentado foi desenvolvido considerando o balanço de mais de um dos óxidos da escória, de modo a distribuir os erros analíticos e aumentar a precisão das previsões, com os dados disponíveis.

\section{MATERIAIS E MÉTODOS}

Para o desenvolvimento do modelo foram acompanhadas 30 corridas de diferentes aços no FEA e no forno panela, registrando-se todos os materiais adicionados a panela a partir do vazamento do FEA e obtendo-se amostras e analisando as escórias do FEA e do FP e a composição química do aço vazado do FEA e no final do refino no FP. As amostras de escória e as amostras de aço foram analisadas por fluorescência de Raios-X em espectrômetros calibrados para os elementos em questão. Os teores de carbono foram determinados, também, por combustão e, quando necessário, teores de oxigênio foram medidos por células eletrolíticas descartáveis. 


\section{FORMULAÇÃO DO MODELO}

O modelo foi formulado como um balanço de massa de todos os elementos relevantes, presentes na panela, desde o vazamento do FEA. Pela conservação da massa dos elementos, considerou-se que os elementos, uma vez adicionados ao volume de controle (a panela) estarão presentes de uma entre duas formas: como parte do aço líquido ou como, óxidos, como parte da escória (o enxofre não foi considerado no balanço). Assumiu-se que a perda por gases (CO, acetileno) e por poeiras, a partir do vazamento do FEA, pode ser desprezada.

As principais entradas consideradas no modelo foram: aço vazado do FEA, escória transferida ou "passante" do FEA para a panela, adições de ferro-ligas, adições de cal calcítica, fluorita, escória sintética, areia de vedação do canal de vazamento do FEA ("chamote").

As principais saídas consideradas são: a escória do FP e o aço liberado para o lingotamento.

Neste modelo, há duas quantidades desconhecidas: o peso de escória "passante" do FEA e o peso de escória final no FP. O modelo visa a calcular, através do balanço de massa dos elementos $\mathrm{Al}, \mathrm{Si}, \mathrm{Ca}, \mathrm{Mn}$ e $\mathrm{Cr}$ estas duas quantidades, simultaneamente.

A seguir é apresentada a implementação do modelo e são discutidas suas principais características e os resultados obtidos com o emprego do modelo.

\subsection{Dados de Entrada do Modelo}

Para a montagem do modelo foram coletadas as composições químicas de todos os insumos usados no vazamento do FEA e no FP. Caso ocorram mudanças de especificação dos insumos os valores devem ser ajustados, no modelo, para considerar estas mudanças.

Para cada corrida, o usuário deve introduzir as seguintes informações (isto pode, evidentemente, ser feito pelo sistema de controle e automação da aciaria). As principais informações são: composições químicas das escórias final do FEA e de saída do FP, composição química e peso do aço vazado no FEA e composição química e peso do aço que chega ao lingotamento, assim como o peso de todas as adições de insumos realizadas desde o vazamento do FEA até a liberação da panela para o lingotamento.

A Figura 1 apresenta um exemplo da planilha do modelo preenchida com os dados de uma corrida hipotética. Os dados a serem preenchidos estão destacados em vermelho. 


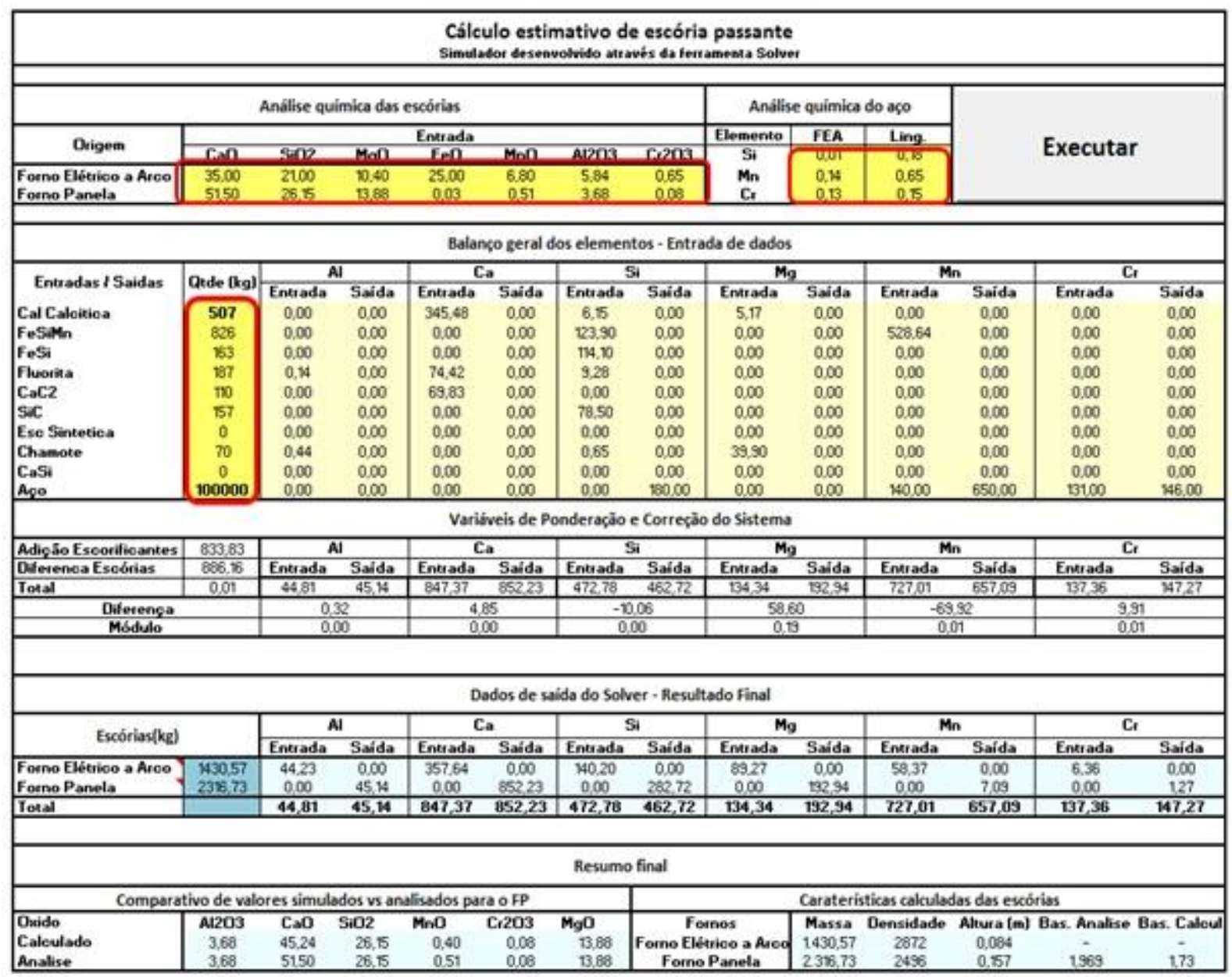

Figura 1. Exemplo dos dados de entrada no modelo de balanço para cálculo de peso de escória.

Observando-se a planilha nota-se que todas as entradas e saídas são calculadas em termos dos elementos químicos, já que estas são as espécies que efetivamente se conservam, na aciaria.

\subsection{Solução do Balanço de Massa pelo Modelo}

Após o preenchimento dos dados, o modelo calcula a diferença entre as quantidades de entrada e saída de cada elemento, e as apresenta na seção da planilha chamada "variáveis de ponderação e correção do sistema", como apresentado na Figura 2. Para o elemento cálcio, por exemplo, a quantidade total de entrada, sem considerar o peso de escória passante do FEA, é de aproximadamente $489,73 \mathrm{~kg}$, neste exemplo. Como o modelo ainda não buscou a solução do balanço de massa (isto é, ainda não existem pesos definidos para as duas escórias, passante e final) este valor indica a quantidade de cálcio que foi adicionada a corrida, apenas, através de diferentes insumos. 


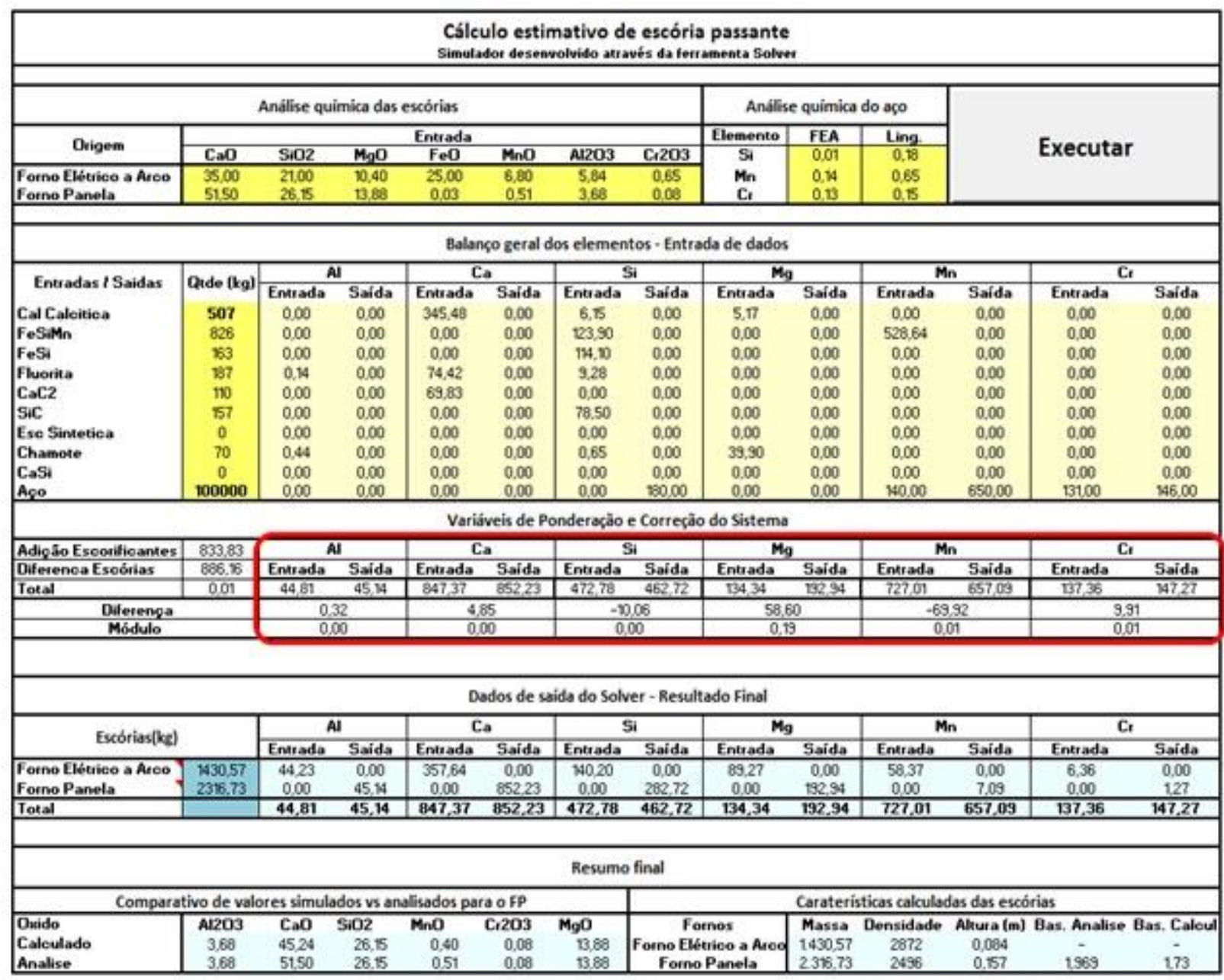

Figura 2. As diferenças entre pesos de entrada e saída de cada elemento estão indicadas na planilha deste exemplo, na região "variáveis de ponderação e correção do sistema".

Para obter uma solução válida para o balanço de massa o modelo busca atender a condição de igualdade entre massa de entrada e de saída para os elementos escolhidos, por exemplo, $\mathrm{Al}, \mathrm{Ca}, \mathrm{Si}, \mathrm{Mn}$ e $\mathrm{Cr}$. Desta forma, observou-se que os erros e incertezas das análises químicas, especialmente das escórias, são diluídos e a solução do modelo se aproxima da solução correta, ideal, fossem todos os pesos conhecidos precisamente. Assim, é aplicada uma rotina de mínimos quadrados para, variando os pesos da escória passante e da escória final no FP, minimizar o somatório apresentado na Equação 1. A Equação 1 expressa o balanço quando um número $n$, qualquer, de elementos, são considerados significativos para a rotina de balanço de massa, e não apenas os 4 elementos citados acima.

$$
\sum_{i=1}^{n}\left(\frac{M_{1, E}-M_{1, S}}{M_{1, E}}\right)^{2}+\left(\frac{M_{2, E}-M_{2, S}}{M_{1, E}}\right)^{2}+\cdots\left(\frac{M_{n, E}-M_{n, S}}{M_{n, E}}\right)^{2}=\text { mínimo }
$$

$\mathrm{Na}$ Equação 1, os subscritos de 1 a $\mathrm{n}$ representam os elementos químicos considerados relevantes para o balanço de massa, $M$, representa a massa e os subscritos E e S identificam, respectivamente, as massas de Entrada e Saída no sistema. É importante observar que todas as diferenças de massa foram 
normalizadas em relação a massa de entrada do respectivo elemento, de modo a que os erros quadrados, da massa de cada elemento, tivessem importância relativa equivalente na minimização do erro total.

O resultado do somatório se encontra na mesma seção e é representado pelo total, conforme é mostrado na Figura 3.

\begin{tabular}{|c|c|c|c|c|c|c|c|c|c|c|c|c|c|}
\hline \multicolumn{14}{|c|}{$\begin{array}{l}\text { Cáleulo estimativo de escória passante } \\
\text { simulador desenvolvido atr wớs đa lentamenta solver }\end{array}$} \\
\hline \multicolumn{8}{|c|}{ Análise quimica das escórias } & \multicolumn{3}{|c|}{ Análise quimica do aşo } & \multirow{4}{*}{\multicolumn{3}{|c|}{ Executar }} \\
\hline \multirow{2}{*}{ Origem } & \multicolumn{7}{|c|}{ Entrada } & \multirow{3}{*}{\begin{tabular}{c|} 
Elemeno \\
Si \\
$\mathrm{Mn}_{\mathrm{n}}$ \\
$\mathrm{C}_{\mathrm{r}}$ \\
\end{tabular}} & \multirow{2}{*}{\begin{tabular}{|l|} 
FEA \\
0.01
\end{tabular}} & \multirow{3}{*}{\begin{tabular}{|c|} 
Ling \\
0.83 \\
0.65 \\
0.85 \\
\end{tabular}} & & & \\
\hline & $\mathrm{CaO}$ & 502 & $\mathrm{MgO}$ & $\mathrm{FeD}$ & $\mathrm{MnO}$ & Al2003 & Cr203 & & & & & & \\
\hline $\begin{array}{l}\text { Forno Elétrico a Arco } \\
\text { Fomo Panela }\end{array}$ & $\begin{array}{l}35,00 \\
5150\end{array}$ & $\begin{array}{l}2100 \\
26,15 \\
\end{array}$ & $\begin{array}{l}10.40 \\
13.89 \\
\end{array}$ & $\begin{array}{l}25.00 \\
0.03 \\
\end{array}$ & $\begin{array}{l}6.80 \\
0.51 \\
\end{array}$ & $\begin{array}{l}5.84 \\
3.68 \\
\end{array}$ & $\begin{array}{l}0.65 \\
0.08 \\
\end{array}$ & & $\begin{array}{l}0.14 \\
0.13\end{array}$ & & & & \\
\hline \multicolumn{14}{|c|}{ Ealanço geral dos elementos - Entrada de dados } \\
\hline \multirow{2}{*}{ Entradas $t$ Saidas } & Dtde $[\mathrm{kq}]$ & \multicolumn{2}{|c|}{ Al } & \multicolumn{2}{|c|}{$\mathrm{Ca}$} & \multicolumn{2}{|c|}{ St } & \multicolumn{2}{|c|}{$M_{9}$} & \multicolumn{2}{|c|}{$M n$} & \multicolumn{2}{|l|}{$\mathrm{CI}_{\mathrm{C}}$} \\
\hline & Choe $(\mathrm{kg})$ & Entrada & Saída & Entrada & Saida & Entrada & Saida & Entrada & Saida & Entrada & Saida & Entrada & Saída \\
\hline Cal Caloitica & 507 & 0.00 & 0.00 & 345.48 & 0.00 & 6.75 & 0.00 & $5 . \pi$ & 0.00 & 0.00 & 0.00 & 0.00 & 0.00 \\
\hline FeSiMn & 826 & 0,00 & 0,00 & 0,00 & 0,00 & 123,90 & 0,00 & 0,00 & 0,00 & 528,64 & 0.00 & 0,00 & 0,00 \\
\hline FeSi & 263 & 0,00 & 0.00 & 0.00 & 0.00 & $124 . x$ & 0.00 & 0.00 & 0.00 & 0,00 & 0.00 & 0,00 & 0,00 \\
\hline Fluoeita & 37 & 0,4 & 0,00 & 74,42 & 0,00 & 9,28 & 0,00 & 0,00 & 0.00 & 0,00 & 0,00 & 0,00 & 0,00 \\
\hline $\mathrm{CaC2}$ & no & 0.00 & 0.00 & 69.83 & 0.00 & 0.00 & 0.00 & 0.00 & 0,00 & 0.00 & 0.00 & 0.00 & 0.00 \\
\hline $\operatorname{sic}$ & $\$ 57$ & 0,00 & 0.00 & 0.00 & 0,00 & 78.50 & 0.00 & 0.00 & 0,0 & 0.00 & 0.00 & 0.00 & 000 \\
\hline Eso Sintetica & 0 & 0.00 & 0.00 & 0.00 & 0.00 & 0.00 & 0.00 & 0.00 & 0.00 & 0.00 & 0.00 & 0.00 & 0.00 \\
\hline Chamote & 70 & 0.44 & 0.00 & 0.00 & 0,00 & 0.65 & 0,00 & 39.90 & 0.00 & 0,00 & 0,00 & 0.00 & 0,00 \\
\hline CaSi & 0 & 0.00 & 0.00 & 0.00 & 0.00 & 0.00 & 0.00 & 0,00 & 0,00 & 0.00 & 0.00 & 0.00 & 0.00 \\
\hline Age & 100000 & 0.00 & 0.00 & 0.00 & 0.00 & 0,0 & 160,00 & 0.00 & 0,00 & 140.00 & 650.00 & 13100 & 145.00 \\
\hline \multicolumn{14}{|c|}{ Variäveis de Ponderaşăo e Correçào do Sistema } \\
\hline Adiq Lo Escorificeant. & 93383 & \multicolumn{2}{|c|}{ Al } & \multicolumn{2}{|c|}{$\mathrm{Ca}$} & \multicolumn{2}{|c|}{ \$i } & \multicolumn{2}{|c|}{$\mathrm{Mg}_{9}$} & \multicolumn{2}{|c|}{$M n$} & \multicolumn{2}{|l|}{$\mathrm{Cl}_{\mathrm{f}}$} \\
\hline Diferenoa Escórias & 86.15 & Entrada & Saida & Entrada & Saida & Entrada & Saida & Entrada & Saida & Entrada & Saida & Entrada & Saida \\
\hline Tetal & 0.01 & 44.81 & 45,14 & 847,37 & 852.23 & 472,78 & 462.72 & 13434 & 192.94 & 727.01 & 657,09 & 137,36 & 197227 \\
\hline \multirow{2}{*}{\multicolumn{2}{|c|}{$\begin{array}{l}\text { Difetençáa } \\
\text { Módulo }\end{array}$}} & & 32 & & $8 \mathrm{SS}$ & & 306 & 58 & & -69 & $\$ 2$ & 99 & \\
\hline & & & 00 & & 00 & & 00 & 0.19 & & 0.0 & 9 & 0.0 & \\
\hline & & & & & ados de sa & ida do Solv & ver - Result & ado Final & & & & & \\
\hline fscóriastkel & & & al & & $a$ & & 54 & $\mathrm{Mg}_{9}$ & & Mr & & $\mathrm{Cr}$ & \\
\hline Escorias(kg) & & Entrada & Saida & Entrada & Saida & Entrada & Saida & Entaada & Saida & Entrada & Saida & Entrada & Saida \\
\hline $\begin{array}{l}\text { Foino Elétrico a Ateo } \\
\text { Famo Panela }\end{array}$ & $\begin{array}{l}1430,57 \\
2315,73\end{array}$ & $\begin{array}{l}44.23 \\
0.00\end{array}$ & $\begin{array}{l}0,00 \\
45,44\end{array}$ & $\begin{array}{c}357,64 \\
0.00\end{array}$ & $\begin{array}{c}0,00 \\
852.23\end{array}$ & $\begin{array}{c}140.20 \\
0.00\end{array}$ & $\begin{array}{c}0,00 \\
282,72\end{array}$ & $\begin{array}{l}89.27 \\
0.00\end{array}$ & $\begin{array}{c}0.00 \\
192.94\end{array}$ & $\begin{array}{l}58,37 \\
0,00\end{array}$ & $\begin{array}{l}0,00 \\
7,09\end{array}$ & $\begin{array}{l}6,36 \\
0.00\end{array}$ & $\begin{array}{l}0.00 \\
127\end{array}$ \\
\hline Total & & 44.81 & 45.14 & 847,37 & 052.23 & 472,78 & 462,72 & 134.34 & 192,94 & 727,01 & 657,09 & 107,36 & 147.27 \\
\hline & & & & & & Resumo & final & & & & & & \\
\hline Compara & tivo de valo & res simula & ados vs an & alisados pa & raofp & & & & Carateris & sticas calculad & las das escó & grias & \\
\hline Oxido & A1203 & $\mathrm{C}=0$ & 5402 & $\mathrm{MnO}$ & $\mathrm{C} 203$ & $\mathrm{MgO}$ & & nos & Massa & Densidade & Altura (m) & Bas. Analise & Bas. Caloud \\
\hline Caloulado & 3.68 & 45.24 & 26,75 & 0.40 & 0.00 & 1380 & Foune Elé & trico a Areed & 1430,57 & 2872 & 0.064 & - & - \\
\hline Analise & 3.68 & 5150 & 26,5 & 0.51 & 0,08 & 13.86 & Foine & Panela & 2383,73 & 2496 & 0,57 & 1969 & 1,73 \\
\hline
\end{tabular}

Figura 3. Somatório das Diferenças

A minimização do valor total corresponde a uma tentativa, através da ferramenta solver, de encontrar valores que façam com que as diferenças dos elementos sejam as menores possíveis. Estes valores que o solver busca para fechar o balanço são, respectivamente, a escória passante do FEA e a geração total do FP.

\subsection{Restrições Aplicadas a Solução Matemática}

Durante a construção do modelo, percebeu-se a necessidade de inserir algumas restrições, por exemplo, para os valores de pesos de escória calculados. Para os valores do peso de escória passante, por exemplo, foi estabelecida a restrição deste peso dentro de um intervalo específico.

Este intervalo foi determinado baseado em medições da altura da escória na panela Através da equação proposta por Turkdogan, em [5], para descrever a densidade 
das escórias de aciaria (Equação 2), confirmou-se que os valores de massa de escória estavam todos, efetivamente, dentro deste intervalo.

$$
\rho_{\text {slag }}=2490 \mathrm{~kg} / \mathrm{m}^{3} \times 12\left(\% \mathrm{FeO}_{\text {slag }}+\% \mathrm{MnO}_{\text {slag }}\right)
$$

Outra restrição imposta a solução do modelo foi o valor diferença de massa entre as escórias do FEA e do FP. Em função das adições realizadas, especialmente cal, foi definida uma diferença mínima entre estas duas massas como restrição à solução do modelo.

Todas as premissas e condições restritivas são mostradas no balanço já calculado, bem como adições de escorificantes e diferenças de escórias.

\begin{tabular}{|c|c|c|c|c|c|c|c|c|c|c|c|c|c|}
\hline \multicolumn{14}{|c|}{$\begin{array}{l}\text { Cálculo estimativo de escória passante } \\
\text { simulados desenvolvido arravis da ferramenta Solver }\end{array}$} \\
\hline \multicolumn{8}{|c|}{ Anisse quimica das escórias } & \multicolumn{3}{|c|}{ Anslise quimica do aco } & \multirow{4}{*}{\multicolumn{3}{|c|}{ Executar }} \\
\hline \multirow{2}{*}{ Onigem } & \multicolumn{7}{|c|}{ Entrads } & \multirow{3}{*}{\begin{tabular}{|c|} 
Elemento \\
Sin \\
$\mathrm{Mn}$ \\
$\mathrm{C}$ \\
\end{tabular}} & \multirow{2}{*}{\begin{tabular}{|l} 
FEA \\
0,01
\end{tabular}} & \multirow{3}{*}{\begin{tabular}{|c|} 
I ing \\
0,75 \\
0,65 \\
0,75 \\
\end{tabular}} & & & \\
\hline & $\mathrm{CaO}$ & $\mathrm{siO} 02$ & $\mathrm{MgO}$ & $1 \in 0$ & $\mathrm{MnO}$ & A2OS3 & Cr203 & & & & & & \\
\hline $\begin{array}{l}\text { Foeno Elétioe a Aleo } \\
\text { Forno Panela }\end{array}$ & $\begin{array}{l}35,00 \\
5150 \\
\end{array}$ & $\begin{array}{l}21,00 \\
28,15 \\
\end{array}$ & $\begin{array}{l}10,40 \\
13,89 \\
\end{array}$ & $\begin{array}{r}25,00 \\
0,03 \\
\end{array}$ & $\begin{array}{l}6.60 \\
0.51 \\
\end{array}$ & $\begin{array}{l}5.84 \\
3.88 \\
\end{array}$ & $\begin{array}{l}0.65 \\
0.08 \\
\end{array}$ & & $\begin{array}{l}0.14 \\
0.13 \\
\end{array}$ & & & & \\
\hline \multicolumn{14}{|c|}{ Balanço geral dos elementos - Entrada de dados } \\
\hline \multirow{2}{*}{ Entradas / Saidas } & & \multicolumn{2}{|c|}{ Al } & \multicolumn{2}{|c|}{$\mathrm{Ca}$} & \multicolumn{2}{|c|}{ Si } & \multicolumn{2}{|c|}{$\mathrm{Mg}_{\mathrm{g}}$} & \multicolumn{2}{|c|}{$M n$} & \multicolumn{2}{|l|}{$\mathrm{C}$} \\
\hline & Qide $(\mathrm{kg})$ & Entrada & Salda & Entrada & Saida & Entrada & Saida & Entiada & Saida & Entrada & Saida & Entuada & Saida \\
\hline Cal Calcitica & 507 & 0.00 & 0,00 & 345.48 & 0.00 & 6.15 & 0.00 & 5.17 & 0.00 & 0.00 & 0.00 & 0.00 & 0,00 \\
\hline Fesimn & 808 & 0.00 & 0.00 & 0.00 & 0.00 & 23.90 & 0.00 & 0,00 & 0.00 & 528,64 & 0.00 & 0.00 & 0.00 \\
\hline FeSi & 263 & 0.00 & 0,00 & 0,00 & 0,00 & $7 \mathrm{~N}, 10$ & 0,00 & 0,00 & 0,00 & 0,00 & 0,00 & 0.00 & 0,00 \\
\hline Fhootina & 207 & $0, \mathrm{~m}$ & 0.00 & 74,42 & 0,00 & 9200 & 0.00 & 0,00 & 0,00 & 0,00 & 0,00 & 0,00 & 0,00 \\
\hline $\mathrm{CaC2}$ & $m$ & 0.00 & 0.00 & 69.83 & 0.00 & 0.00 & 0,00 & 0,00 & 0.00 & 0.00 & 0.00 & 0.00 & 0,00 \\
\hline Sic & 157 & 0.00 & 0,00 & 0.00 & 0.00 & 78.50 & 0.00 & 0.00 & 0.00 & 0.00 & 0.00 & 0.00 & $0, \infty$ \\
\hline Eso Sintetioa & 0 & 0.00 & 0.00 & 0.00 & 0.00 & 0.00 & 0.00 & 0.00 & 0.00 & 0.00 & 0,00 & 0.00 & 0.00 \\
\hline Chamote & 70 & 0,44 & 0.00 & 0.00 & 0,00 & 0.65 & 0.00 & 39.90 & 0,00 & 0,00 & 0.00 & 0.00 & 0.00 \\
\hline CaSi & 0 & 0.00 & 0,00 & 0,00 & 0,00 & 0.00 & $0, \infty$ & 0.00 & 0,00 & 0,00 & 0,00 & 0.00 & $0, \infty$ \\
\hline Apo & 100000 & 0.00 & 0,00 & 0,00 & 0.00 & 0,00 & 100,00 & 0,00 & 0,00 & 140,00 & 650,00 & 13100 & $\$ 6,00$ \\
\hline \multicolumn{14}{|c|}{ Variäveis de Ponderaç̆o e Corregào do Sistema } \\
\hline Adigăo Escorilicantes & 833.83 & \multicolumn{2}{|c|}{ Al } & \multicolumn{2}{|c|}{$\mathrm{Ca}$} & \multicolumn{2}{|c|}{\begin{tabular}{|c|} 
Si \\
\end{tabular}} & \multicolumn{2}{|c|}{$\mathrm{Mg}$} & \multicolumn{2}{|c|}{$M n$} & \multicolumn{2}{|l|}{ G } \\
\hline Diterenoa Escóntias & $868.8 \%$ & Entrada & Salda & Entrada & Saids & Entrada & Salda & Entiada & Saida & Entrada & Saida & Entrada & Saida \\
\hline Total & 0.01 & 44.51 & 45.14 & 647,37 & 65223 & 472.78 & 46272 & 134,34 & 75254 & 72701 & 657,09 & 13735 & 97.27 \\
\hline \multirow{2}{*}{\multicolumn{2}{|c|}{$\begin{array}{l}\text { Diferenga } \\
\text { Módulo }\end{array}$}} & & 32 & & 85 & & 2.08 & 58.6 & & -69 & 98 & 99 & 91 \\
\hline & & & 00 & & $\infty 0$ & 0 & 00 & $0, \pi$ & & 0.0 & 01 & 00 & 우 \\
\hline & & & & & lades de se & aide do Solv & ver-Result & tado Final & & & & & \\
\hline & & & al & & Ca & 5 & 5 & $\mathrm{Mg}$ & & $\mathrm{Mn}$ & In & $\mathrm{C}$ & 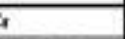 \\
\hline Escorias $(\mathrm{kg})$ & & Entioda & Saida & Entrada & Saida & Entrada & Saída & Entuada & Saida & Entrada & Saida & Enttada & Saida \\
\hline $\begin{array}{l}\text { Forno Elétricos a Arco } \\
\text { Forno Pannela }\end{array}$ & $\begin{array}{l}1490.57 \\
232573\end{array}$ & $\begin{array}{c}44,23 \\
0,00\end{array}$ & $\begin{array}{l}0,00 \\
45.14\end{array}$ & $\begin{array}{c}357,64 \\
0,00\end{array}$ & $\begin{array}{c}0,00 \\
852,23\end{array}$ & $\begin{array}{c}140,20 \\
0,00\end{array}$ & $\begin{array}{c}0,00 \\
28272\end{array}$ & $\begin{array}{c}89,27 \\
0,00\end{array}$ & 0,00 & $\begin{array}{c}58,37 \\
0.00\end{array}$ & $\begin{array}{l}0,00 \\
7,09\end{array}$ & $\begin{array}{l}6,36 \\
0,00\end{array}$ & $\begin{array}{l}0.00 \\
127\end{array}$ \\
\hline Total & & 44.81 & 45.14 & 847,37 & 852,23 & 472.78 & 462,72 & 134,34 & 192,94 & 727.01 & 657.09 & 137,36 & 147.27 \\
\hline & & & & & & Resumo & final & & & & & & \\
\hline Compara & tivo de val & ores simul & ados vs an & alisados pe & ra o FP & & & & Carateris & isticas calculad & das das escó & órias & \\
\hline Onido & Al203 & $\mathrm{CaO}$ & $\operatorname{sic2}$ & $\mathrm{MnO}$ & C.203 & $\mathrm{MgO}$ & & anos & Massa & Densidade & Altura (m) & Bas, Analise & Bas, Caloul \\
\hline Caleulado & 3.68 & 45.24 & 26,5 & 0.40 & 0.06 & 13,86 & Forne Ele & trieo a Arod & 1430.57 & 2872 & 0.004 & the & - \\
\hline Analise & 3.68 & 5150 & 26,15 & 0.51 & 0,06 & 1389 & Fome & Panela & $2.315,73$ & 2436 & 0.57 & 1369 & 1.73 \\
\hline
\end{tabular}

Figura 4. Restrições impostas em relação às quantidades de escorificantes adicionados e as massas de escória calculadas.

\subsection{Dados de Saída e Resultado Final do Modelo}

Os dados de saída são apresentados na seção Dados de Saída da planilha, como mostra a Figura 5. Nesta seção são mostrados os resultados calculados das quantidades de escória do FEA e do FP além das quantidades de entrada e saída de cada elemento. 
Na seção Resumo Final, da planilha, é mostrada a comparação entre as análises de escória calculada e analisada no FP. É mostrada também a altura de escória na panela estimada através da equação da densidade (Equação 2).

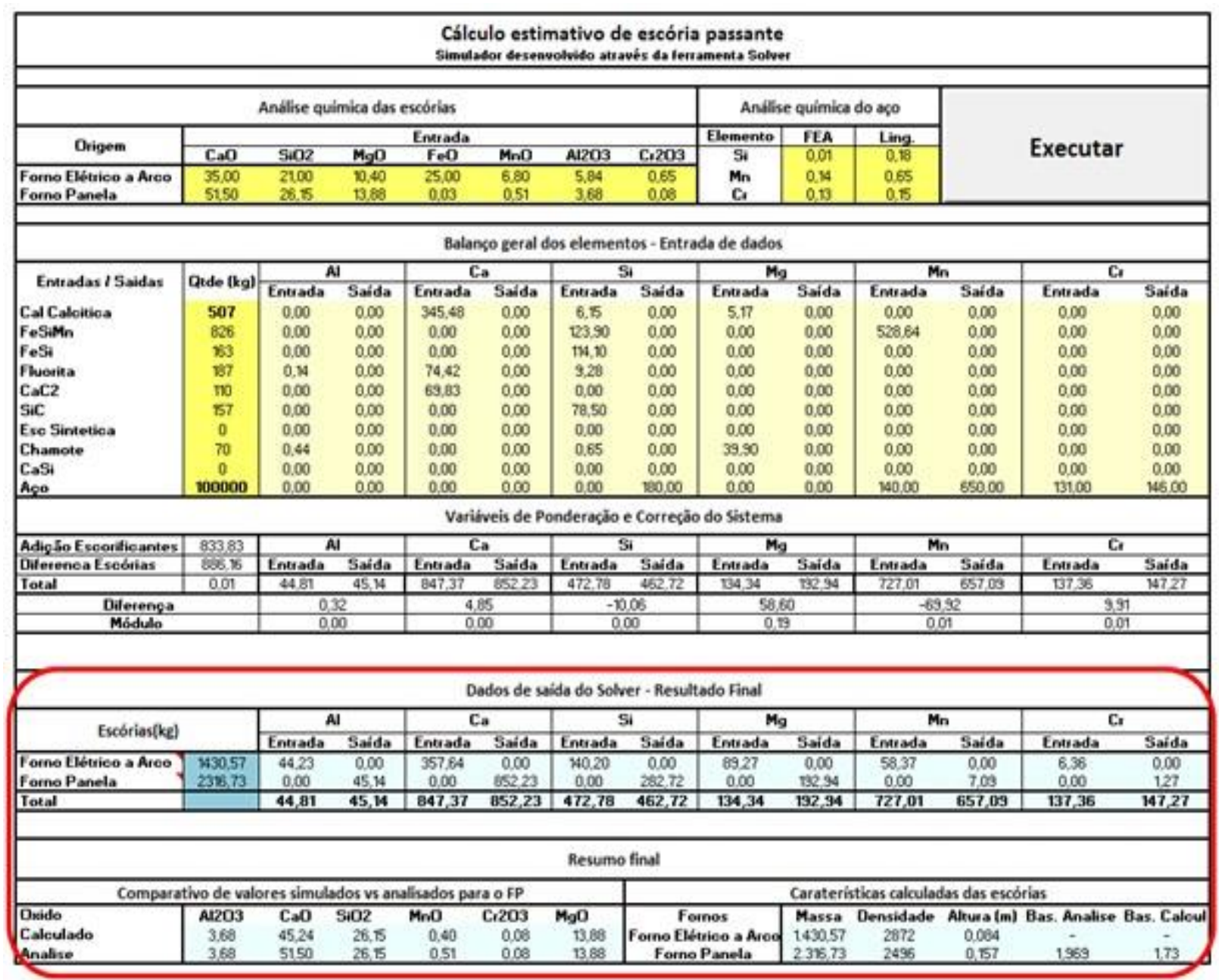

Figura 5. Apresentação do Dados de Saída e Resultado Final.

Segundo os cálculos mostrados na Figura 5, as quantidades de escória do FEA e do FP são 1430 e $2316 \mathrm{Kg}$ respectivamente. A quantidade de escória passante do FEA representaria uma altura de aproximadamente $8,4 \mathrm{~cm}$, na panela, e a escória no fim do processamento do FP teria cerca de $16 \mathrm{~cm}$ de espessura.

A assertividade do modelo foi verificada por medições de espessura de camada de escória em algumas corridas, convertidas em massa através da expressão da densidade da escória da Equação 2. Adicionalmente, o fornecedor de escória sintética realizou balanços de massa independentes. Em ambos os casos os resultados do modelo foram bem próximos das medidas e dos resultados do balanço de massa alternativo.

\section{CONCLUSÃO}

O modelo apresentado permite, de uma maneira rápida e simples, calcular as quantidades de escória passante do FEA e presente no final do processamento do FP. Ao contrário de outros métodos que exigem maiores recursos financeiros e/ou 
são baseados em formulações matemáticas mais complexas, a implantação do modelo proposto não requer investimento elevado, já que utiliza recursos utilizados pela maioria das empresas (planilhas eletrônicas) e se baseia em equações e condições relativamente simples e em medidas e análises que são, frequentemente, realizadas em todas as aciarias, mesmo quando não existe tal modelo.

Embora não seja possível alcançar precisão total, em função dos vários erros inerentes aos processos de análise, pesagem etc., os valores obtidos pelos cálculos apresentaram-se condizentes com os valores aferidos da altura de escória na panela antes e depois do processamento no FP

Assim, o modelo apresentado tem-se mostrado uma ferramenta adicional no controle de processos durante o refino secundário, sendo possível, através de sua utilização, efetuar as correções necessárias para se obter uma escória com as características desejáveis no refino secundário.

\section{Agradecimentos}

Os autores agradecem a GERDAU pelo apoio e pela oportunidade de realização deste trabalho e pela autorização para sua publicação.

\section{REFERÊNCIAS}

1 Ban-ya S. Preface- Special Issue on Physics and Chemistry of Molten Slags. ISIJ International. 1993;33(1):1.

2 E Pretorious, H Oltmann. Fundamentals of the EAF Process. LWB Refractories; 2011, [acesso em 04 abr 2016] disponível em http://etech.Iwbref.com/Downloads/ Theory/ Fundamentals\%20of\%20the\%20EAF\%20Process.pdf.

3 Gaye H, Gatellier C, Nadif M, Riboud PV, Saleil J, Faral M. Reaction metal-latier et controle de la composition des inclusions residuelles en metallurgie secondaire. Revue Metallurgie CIT. 1987;(Novembre):759-71.

4 Suito $\mathrm{H}$, Inoue $\mathrm{R}$. Thermodynamics on control of inclusions composition in ultra-clean steels. ISIJ International. 1996;36(5):528-36.

5 Fruehan R, editor. Making, Shaping, and Treating of Steel, Steelmaking And Refining Volume. 11th Edition. Pittsburgh PA: AISE Steel Foundation; 1998. 